\title{
Expression profiles of mTOR pathway proteins in porocarcinoma: A provisional immunohistochemical study
}

\author{
MITSUAKI ISHIDA and HIDETOSHI OKABE \\ Department of Clinical Laboratory Medicine and Division of Diagnostic Pathology, \\ Shiga University of Medical Science, Otsu, Shiga 520-2192, Japan
}

Received August 23, 2012; Accepted September 28, 2012

DOI: $10.3892 /$ br.2012.20

\begin{abstract}
Porocarcinoma is a rare skin appendage carcinoma, with a poor prognosis. At present, the recommended treatment of localized porocarcinoma is wide surgical resection. Although anthracyclin-based chemotherapy or combination of 5-fluorouracil (5-FU), taxanes and cisplatin are considered to be the first-line treatment for metastatic or locally-advanced porocarcinoma, this type of tumor is recognized as relatively chemoresistant, and no standard systemic treatment has been established yet. Mammalian target of rapamycin (mTOR) is an important protein involved in carcinogenesis. mTOR phosphorylates the eukaryotic translation initiation factor 4E-binding protein 1 (4E-BP1), and then phosphorylated 4E-BP1 (p4E-BP1) triggers cell cycle progression, cell proliferation and angiogenesis. Therefore, mTOR is believed to be one of the most promising therapeutic targets in various types of carcinomas. However, the expression profiles of mTOR pathway proteins in porocarcinoma have yet to be elucidated. Therefore, we analyzed the expression of mTOR, 4E-BP1 and p4E-BP1 in five cases of porocarcinoma (four invasive and one in situ case) using immunohistochemical methods. mTOR expression was observed in the invasive porocarcinoma cases, but not in the in situ case. 4E-BP1 was expressed in all five cases. p4E-BP1 expression was observed in 3/4 invasive porocarcinoma cases, but not in the in situ case. This preliminary study clearly demonstrated the overexpression of mTOR and its downstream proteins in most of the included invasive porocarcinoma cases. Therefore, mTOR inhibitors could be considered as potential therapeutic modalities for the treatment of metastatic or locally-advanced porocarcinoma.
\end{abstract}

Correspondence to: Dr Mitsuaki Ishida, Department of Clinical Laboratory Medicine and Division of Diagnostic Pathology, Shiga University of Medical Science, Tsukinowa-cho, Seta, Otsu, Shiga 520-2192, Japan

E-mail: mitsuaki@belle.shiga-med.ac.jp

Key words: mammalian target of rapamycin, eukaryotic translation initiation factor $4 \mathrm{E}$-binding protein 1 , porocarcinoma, skin

\section{Introduction}

Porocarcinoma is a rare skin appendage carcinoma related to the sweat gland duct and is known to be detected in $0.004 \%$ of skin biopsy specimens (1). Its prognosis is not favorable due to the fact that it carries a significant risk of local recurrence, as well as lymph node and distant metastases $(2,3)$. At present, the recommended treatment of localized porocarcinoma is wide surgical resection and in the cases where lymph node metastasis is present, radical lymph node dissection is added. Although anthracylin-based chemotherapy or combination of 5-fluorouracil (5-FU), taxanes and cisplatin are considered to be the first-line treatment for metastatic or locally-advanced porocarcinoma, this type of tumor is recognized as relatively chemoresistant, and no standard systemic treatment exists (4).

Mammalian target of rapamycin (mTOR) is a key protein involved in carcinogenesis and is activated via the phosphatidylinositol-3 kinase (PI3K)/AKT pathway. mTOR phosphorylates the eukaryotic translation initiation factor 4E-binding protein 1 (4E-BP1), and then the phosphorylated 4E-BP1 (p4E-BP1) triggers cell cycle progression, cell proliferation and angiogenesis (5). Therefore, mTOR is believed to be one of the most promising therapeutic targets in various types of carcinomas. Recently, mTOR inhibitors have been demonstrated to prolong the progression-free survival of patients with pancreatic neuroendocrine tumor (6). Moreover, the expression levels of mTOR pathway proteins have been suggested to be predictive markers to gauge response to treatment with mTOR inhibitors (7). However, the expression profiles of mTOR pathway proteins in porocarcinoma have yet to be elucidated. In this preliminary study, we investigated the expression profiles of mTOR, 4E-BP1 and p4E-BP1 in porocarcinoma cases, and discussed whether or not $\mathrm{mTOR}$ inhibitors are suitable candidates for the treatment of porocarcinoma.

\section{Materials and methods}

Expression of mTOR, 4E-BP1 and p4E-BP1 was analyzed in five cases of porocarcinoma (four invasive and one in situ case), using immunohistochemical methods. Immunohistochemical analyses were carried out using an autostainer (XT system Benchmark; Ventana Medical Systems, Tucson, AZ, USA), 


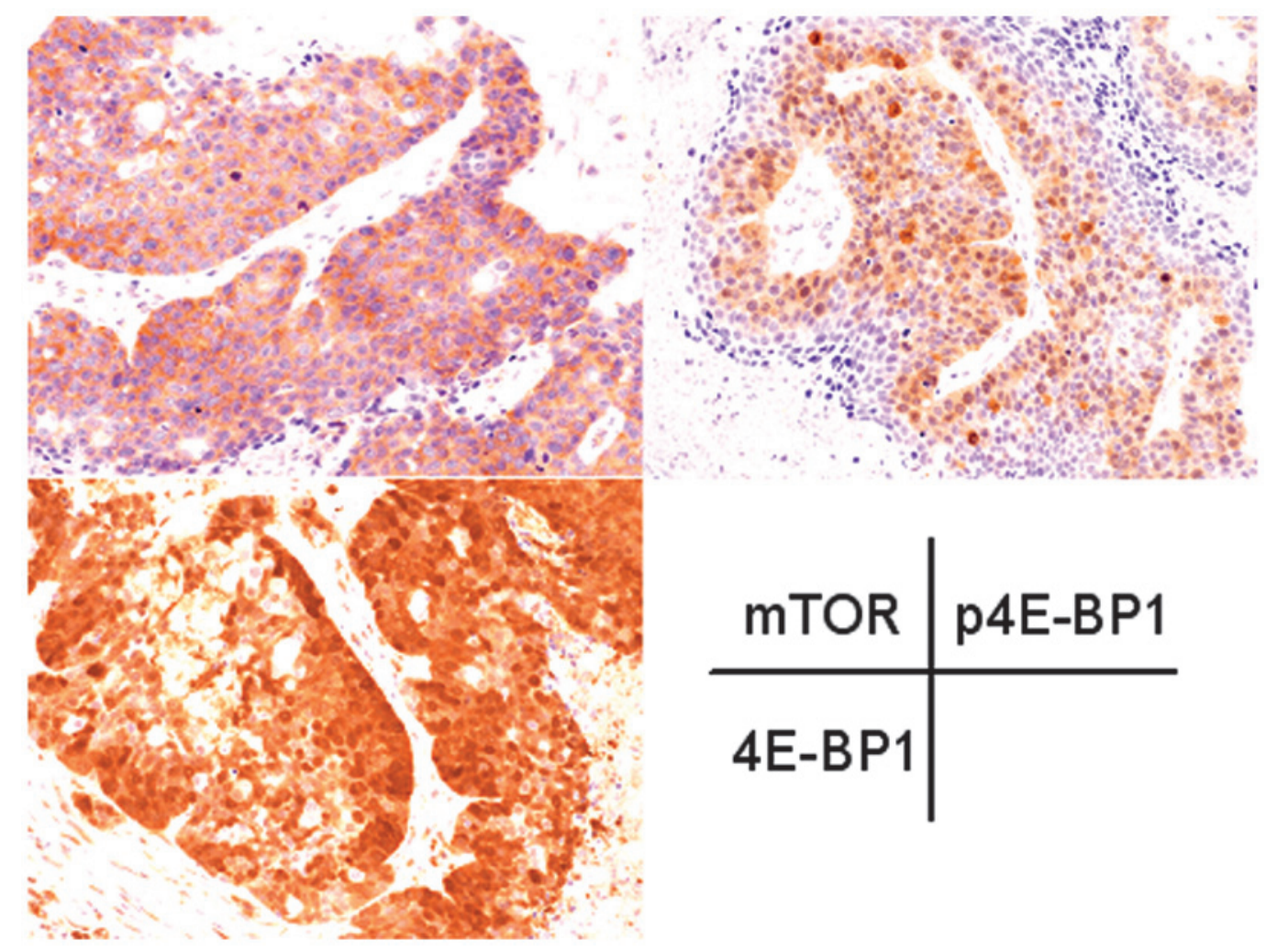

Figure 1. Immunohistochemical staining for mTOR and its downstream proteins in porocarcinoma are shown. mTOR and p4E-BP1 expressed in the cytoplasm of invasive porocarcinoma cells. 4E-BP1 expressed in the cytoplasm of porocarcinoma cells.

according to the manufacturer's instructions. The following primary antibodies were used: a rabbit monoclonal antibody against mTOR (7C10; Cell Signaling Technology, Inc., Danvers, MA, USA), a rabbit monoclonal antibody against 4E-BP1 (53H11; Cell Signaling Technology, Inc.) and a rabbit monoclonal antibody against p4E-BP1 (Thr 37/46) (236B4; Cell Signaling Technology, Inc.).

The immunostaining procedures of these markers were scored using semi-quantitative scoring, as described in a previous study (8).

\section{Results}

Table I shows the immunohistochemical staining results of the mTOR pathway proteins. The expression of mTOR was observed in the invasive porocarcinoma cases, but not in the in situ porocarcinoma case. 4E-BP1 was expressed in the invasive and in situ porocarconima cases. Cytoplasmic p4E-BP1 expression was observed in 3/4 invasive porocarcinoma cases, but not in the in situ porocarcinoma case (Fig. 1).

\section{Discussion}

To the best of our knowledge, this is the first study to investigate the expression profiles of the mTOR pathway proteins in porocarcinoma, and the overexpression of mTOR and its downstream proteins in most of the included invasive porocarcinoma cases was clearly demonstrated. Activation of the mTOR pathway has been suggested to be highly involved in the pathogenesis of extramammary Paget's disease (9), as
Table I. Expression patterns of mTOR pathway proteins in porocarcinoma cases $(n=5)$.

\begin{tabular}{lccc}
\hline & \multicolumn{3}{c}{ mTOR pathway proteins } \\
\cline { 2 - 4 } Porocarcinoma cases & mTOR & 4E-BP1 & p4E-BP1 \\
\hline Invasive $(\mathrm{n}=4)$ & $4 / 4$ & $4 / 4$ & $3 / 4$ \\
In situ $(\mathrm{n}=1)$ & $0 / 1$ & $1 / 1$ & $0 / 1$ \\
\hline
\end{tabular}

mTOR, mammalian target of rapamycin; 4E-BP1, eukaryotic translation initiation factor 4E-binding protein 1; $44 \mathrm{E}-\mathrm{BP} 1$, phosphorylated 4E-BP1.

well as in the carcinogenesis of porocarcinoma, especially during the invasive stage.

Moreover, findings of a previous study demonstrated that treatment with mTOR inhibitors led to growth inhibition and cell cycle arrest in the G1 phase of carcinoma cells (10). The present study clearly demonstrated that the mTOR pathway was activated in most of the included invasive porocarcinoma cases. This finding, together with the findings of previous studies, $(6,10)$ demonstrate that mTOR inhibitors are potential therapeutic modalities for the treatment of metastatic or locally-advanced porocarcinoma. Additional clinicopathological studies as well as clinical trials concerning treatment with mTOR inhibitors in patients with porocarcinoma are required to provide more definite evidence. 


\section{References}

1. Mehregan AH, Hashimoto K and Rahbari H: Eccrine adenocarcinoma. A clinicopathologic study of 35 cases. Arch Dermato 119: 104-114, 1992.

2. Snow SN and Reizner GT: Eccrine porocarcinoma of the face. J Am Acad Dermatol 27: 306-311, 1992.

3. Ishida M, Hotta M, Kushima R and Okabe H: A case of porocarcinoma arising in pigmented hidroacanthoma simplex with multiple lymph node, liver and bone metastases. J Cutan Pathol 38: 227-231, 2011

4. Perez-Garcia J, Morales R, Valverde CM, et al: Eccrine porocarcinoma presenting with scrotal lymphedema: a case report and review of systemic treatment. Ann Oncol 21: 907, 2010.

5. Faivre S, Kroemer G and Raymond E: Current development of mTOR inhibitors as anticancer agents. Nat Rev Drug Discov 5: 671-688, 2006.
6. Yao JC, Shah MH, Ito T, et al: Everolimus for advanced pancreatic neuroendocrine tumors. N Engl J Med 364: 514-523, 2011.

7. Duran I, Kortmansky J, Singh D, et al: A phase II clinical and pharmacodynamic study of temsirolimus in advanced neuroendocrine carcinomas. Br J Cancer 96: 1148-1154, 2006.

8. Darb-Esfahani S, Faggad A, Noske A, et al: Phospho-mTOR and phospho-4EBP1 in endometrial adenocarcinoma: association with stage and grade in vivo and link with response to rapamycin treatment in vitro. J Cancer Res Clin Oncol 135: 933-941, 2009.

9. Chen S, Nakahara T, Uchi H, et al: Immunohistochemical analysis of the mammalian target of rapamycin signaling pathway in extramammary Paget's disease. Br J Dermatol 161: 357-363, 2009.

10. Gao N, Flynn DC, Zhang Z, et al: G1 cell cycle progression and the expression of $\mathrm{G} 1$ cyclins are regulated by $\mathrm{PI} 3 \mathrm{~K} / \mathrm{AKT} / \mathrm{mTOR} / \mathrm{p} 70 \mathrm{~S} 6 \mathrm{~K} 1$ signaling in human ovarian cancer cells. Am J Physiol Cell Physiol 287: C281-C291, 2004. 